\title{
UN ACERCAMIENTO A LA SOCIO-CIBERNÉTICA Y LA INFOÉTICA EN LAS TIC: VALORES ÉTICOS Y DERECHO
}

\author{
AN APPROACH TO SOCIO-CYBERNETICS AND INFOETHICS IN ICT: ETHICAL VALUES \\ AND LAW
}

YARINA AMOROSO FERNÁNDEZ

Universidad de las Ciencias Informáticas

yarinamoroso@gmail.com

RECIBIDO: 08/09/2017

ACEPTADO: $30 / 11 / 2017$

Resumen: El impacto social de las TIC genera problemas éticos, sociales, políticos y jurídicos. El objetivo del presente estudio es apoyar una mirada sistémica que permita comprender mejor y afrontar los referidos problemas desde la Socio-cibernética, la Infoética y el Derecho dado su naturaleza unitaria. La autora considera que si se propicia la interrelación entre estas áreas de las ciencias sociales se pueden encontrar fórmulas que sean portadoras de soluciones que la sociedad está demandando para ordenar un uso de las TIC que favorezca la convivencia civilizada y pacífica en el Ciberespacio, el desarrollo social y el respecto a la dignidad humana.

Palabras clave: Derecho, Cibernética, Ciberespacio, Socio-cibernética, Infoética, impacto social de las TIC.

\begin{abstract}
The social impact of the ICT generates ethical, social, political and juridical problems. With the objective of contributing to a systemic look that allows to understand them better and to face them appropriately intends an approach to the socio-cybernetics, the Infoethics and the Law, given their unitary nature. It is considered that if the interrelation among these areas of the social sciences is propitiated, these can be formulas that became carriers of that kind of solutions that the humanity and the individual are demanding to order the use of the ICT and at the same time to favor peaceful and civilized coexistence in the Cyberspace, social development and human dignity.

Keywords Law, Cybernetics, Cyberspace, Socio-cybernetics, Infoethics, social impact of ICT.
\end{abstract}

\section{A modo de introducción}

Desde los orígenes de la Cibernética se registra la gestación de una sociedad tecnológicamente avanzada que se descubre en estado de evolución y cambios permanentes. También desde ese entonces se evoca un nexo con la Ética y el Derecho porque la humanidad se enfrenta a la selección inmediata de políticas que orienten las prácticas cibernéticas y ordenen el uso social de las tecnologías que hoy conocemos. 
Las Tecnologías de la Información y las Comunicaciones (TIC) constituyen una plasmación instrumental de la Cibernética y mantienen un desarrollo vertiginoso que se distingue por la velocidad de cambios y la diversificación en las maneras de mostrarse.

La convergencia de medios de comunicación y la continua aparición de tecnologías emergentes (Rundle, Conley 2007, Martino 2015) son también rasgos del presente. Se imponen patrones de consumo y sirven a la vez para favorecer una visión emancipadora a partir del uso de las tecnologías.

A la sociedad tecnológicamente avanzada se le ha dado en llamar, tanto por especialistas como por instituciones internacionales, Sociedad de la Información o Sociedad del Conocimiento; Castell (2009) la identifica como Sociedad Red. En coincidencia con Pérez Luño (2014) se reconoce además la existencia del Ciberespacio como un área para la comunicación, el comercio y la participación ciudadana de donde también se evoca nexos con la Ética y el Derecho.

Así, el fenómeno se muestra como un problema complejo y reclama soluciones integradoras. El Ciberespacio es el escenario donde se confrontan actores individuales e institucionales con nuevas situaciones. En ese contexto surgen nuevos roles y se ponen de manifiesto conductas con implicaciones jurídicas, como lo son los derechos: de información y sobre la información. Del mismo modo se manifiestan las relaciones de propiedad; se exigen obligaciones y se comparte responsabilidades.

Es también un espacio en el que se ejerce gobierno y control, se pone en evidencias las hegemonías y se ejercita poder. Con "la nube", además de ser un espacio de gestión de información, se comienza también a materializar actividades computacionales compartidas (Rundle, Conley 2007, Clair 2017).

El empleo de las TIC ofrece la oportunidad de dar nueva vitalidad a los gobiernos pero también es necesario favorecer la participación del ciudadano en la gestación de políticas públicas y en los procesos de gobernanza.

La instauración de soluciones de Gobierno electrónico lleva implícito superar la visión de clientes digitales a ciudadanos, política, económica y socialmente digitales. Estas soluciones tienen que estar destinadas a facilitar la vida de la comunidad. Constituir ahorros en los gastos públicos y permitir el aumento de eficiencia y transparencia (Nahabietián 2013).

Desde finales de la década de los 90 del siglo XX, y abiertamente en estos lustros del siglo XXI, se evidencia que la acelerada velocidad de la evolución tecnológica deja poco tiempo a los decisores, los legisladores y otros sujetos para anticiparse y adsorber los cambios.

En muchas ocasiones, en pleno proceso de decisión legislativa, surge una tecnología disruptiva que impone adaptarse otra vez a la ola de transformación 
que le es consustancial. Este es un reto fundamental para la Ética y también para el Derecho.

En el caso del Derecho, en ocasiones el propio desarrollo tecnológico implica imposición de conductas que encuentran tutela en el ordenamiento y que generalmente responden a intereses de mercado o emporios tecnológicos.

También puede darse el caso que el avance provoque que los decisores no alcancen a comprender que la legislación por sí solo no resuelve el problema.

Otras veces, los resultados legislativos provocan que el impacto se reproduzca a una dimensión diferente o se amplifiquen sus consecuencias, como ha sido el tema de la protección de Datos Personales. Existe una cuarta generación de legislaciones y los problemas persisten porque no alcanzan a atemperarse a las circunstancias tecnológicas.

Por otra parte, las soluciones informáticas generalmente desconocen los principios de protección de datos entre sus requisitos, aunque este panorama debe comenzar a cambiar a partir de asumirse de un modo responsable la filosofía de protección por diseño y desde el diseño.

La evolución, aun discreta, en materia de Protección de Datos Personales es un ejemplo de ello. También existen ejemplos de empleo de la tecnología para el uso inescrupuloso de datos e información que socavan la independencia al tiempo de comprometer la soberanía de los Estados.

De modo que, con frecuencia, sin tenerse todas las respuestas, surgen nuevas interrogantes. En otras ocasiones a causa del avance tecnológico lo que cambia son las preguntas.

Pero hay momentos en que las consecuencias negativas son el resultado de políticas erradas, en virtud de la cuáles se implantan soluciones que fracasan porque se desconocen tradiciones, la inteligencia organizacional, la capacidad social para asumir el cambio o el desarrollo tecnológico local; por solo citar algunas circunstancias.

También, se ha evidenciado que las respuestas no siempre han sido integrales, por lo que se genera brechas que favorecen prácticas inescrupulosas que atentan contra valores de la libertad, la igualdad, la solidaridad, la tolerancia y la responsabilidad compartida. Aumenta la desconfianza y crece la sensación de inseguridad (Pérez Luño 2014).

Pocas veces la introducción tecnológica va acompañada del estudio de impacto desde las ciencias sociales; o mejor aún, articuladas con las áreas de estudios tecnológicos. Por eso soluciones de éxito y buenas prácticas han sido ignoradas. También puede que hayan sido silenciadas al ser referentes válidos para el empoderamiento social y vía de participación democrática en la toma de 
decisiones, como lo fue el caso del Proyecto Cybersyn de Chile (Amoroso 2014).

En el ámbito del impulso de soluciones lo usual es que sean revelados los productos en términos de mercado pero generalmente se encubren los fines del desarrollo tecnológico.

Las soluciones casi siempre quedan sujetas a férreas reglas de propiedad intelectual. Por medio de cláusulas de adhesión se imponen modelos de negocio, que entre otras consecuencias aseguran la dependencia a un mercado o comprometen los procesos de transferencia tecnológica.

En este escenario, el movimiento de Software Libre aún requiere imponerse como alternativa y ser la garantía real para la preconizada soberanía tecnológica.

También avanzan con lentitud los procesos de Acceso Libre y la instauración de políticas de gestión y conservación del Patrimonio Digital, que constituye una garantía de continuidad digital. La base para el acceso a la Memoria del Mundo se instala a muy lento andar.

Es difícil en pocas cuartillas abordar el tema en todo su alcance, pero se asume el reto en el ánimo de utilizar este espacio para motivar el interés por el tema y multiplicar voluntades que permitan integrar áreas de conocimiento social y tecnológico desde la Socio-cibernética, la Infoética y el Derecho.

Se impone superar el estado de preocupación para asumir definitivamente desde las ciencias sociales la actitud de ocupación en la búsqueda de soluciones.

Para ello, es necesario partir del análisis y una sistematización de experiencias prácticas con impacto en el ámbito humano que reconozca en las ciencias sociales una instancia revitalizadora de los valores democráticos e impulsora de una política acorde con las exigencias del presente (Gómez 2006, Pérez Luño 2014).

Se necesita de miradas integradoras y soluciones sistémicas. A través de la Socio-cibernética que deviene de la Cibernética se puede erigir la base teórica primaria para entender el mundo virtual.

Con auxilio de la Infoética que constituye la expresión de voluntad consensual y concertada a partir de la convocatoria de la UNESCO se puede obtener reglas asumibles desde la Ética Aplicada.

El Derecho que es más que el llamado Derecho Informático puede ampliar su capacidad de respuesta con auxilio de la Socio-cibernética y la Infoética.

Se considera que desde estas tres áreas se pueden conjugan respuestas a las interrogantes que hoy plantean los procesos de acción social y desarrollo humano que se gestan de la interacción con las TIC y las tecnologías emergentes (TE). 


\section{La mirada desde las Ciencias Sociales}

La sociedad tecnológicamente avanzada es más dinámica y está sujeta a cambios en menor tiempo. Por eso resulta necesario orientar los esfuerzos a formular un nuevo modelo para su análisis desde las ciencias sociales. Estas deben atender las actuales demandas de variabilidad y complejidad de los sistemas sociales, así como identificar los desafíos del desarrollo social internacional, en cuanto a metas comunes a alcanzar en todas las regiones, y que orienten la acción social global para afianzar las conquistas sociales de la humanidad (Aguado 2006).

La inseguridad ciudadana, la suplantación de la identidad, la invasión a la privacidad y la intimidad, la discriminación, la ineficiencia en la gestión administrativa, la corrupción y la imposibilidad de manipulación eficaz de $n$ volumen de información digital que crece de forma exponencial, son entre otros, algunos de los muchos otros males que aquejan a la sociedad tecnológicamente avanzada. Sus efectos se extienden más allá de los tradicionales límites nacionales y regionales.

Se necesita una nueva perspectiva internacional para ordenar con urgencia los nuevos espacios que son consecuencias de un fenómeno de dimensiones culturales y que evoca cambio de paradigmas porque inciden en la organización y las conductas sociales.

Sus efectos repercuten por doquier, porque los trastornos se globalizan y se afianzan en donde existen las condiciones para su reproducción y mutan haciendo un continuo de dolencias sociales. También se amplifican males ancestrales, como es el caso de la brecha digital, que es una expresión más de la brecha social, a causa de desigualdades en materia de justicia social y del derecho al desarrollo. Estas son realidades que por decenios aquejan a la humanidad en muchas partes del mundo y antecede a la Era Digital.

La información que a diario recibimos pone al descubierto, o al menos llama la atención, que la sociedad no encuentra respuestas eficientes desde las áreas funcionales de las ciencias sociales. Quienes se dedican a la investigación afirman que se impacta tanto a postulados teóricos como en la interpretación de resultados empíricos (Marcuello 2006).

Por ello, cada vez se acepta, de manera más amplia, que la ciencia debe generar conocimientos que puedan ser traducidos en nuevos conceptos y en aplicaciones prácticas eficientes. Relacionado con lo anterior, se afirma que las ciencias sociales deben proporcionar un mapa orientador y las herramientas necesarias para actuar en nuestro mundo pero hay que definir cómo hacerlo.

Se considera que un primer paso es desarrollar, por parte de los científicos sociales, una aptitud y métodos de investigación y análisis propios acorde al 
carácter sistémico de la sociedad, y propender a abordar los problemas por grupos multidisciplinarios. Todo esto con el fin de abordar los problemas desde una mirada intra, inter y transdiciplinar que incluya referentes teóricos y la aplicación de modelos cibernéticos.

Se afirma que la Socio-cibernética puede aportar una vista más dinámica y completa, tanto para las actividades profesionales, como para las preocupaciones teóricas de las ciencias sociales; toda vez que ofrece un promisorio avance en los estudios de organizaciones y sistemas complejos (Gómez 2006).

Asumir la Socio-cibernética de conjunto con la Infoética ayuda a superar una limitada y perjudicial visión tecno-céntrica de los procesos de informatización y nos permite obtener una acertada visión socio-técnica de las aplicaciones tecnológicas que se instalan. Supone además comprender la naturaleza, fines y esencia sistémica del Derecho, y posesionarlo en perspectiva de interoperabilidad (Saarenpää 2015).

Para aproximarse al tema se requiere identificar orígenes y aprehender los conceptos claves, así como atar los elementos de relación y aplicación de las ciencias sociales, sin desconocer el desarrollo científico general que se encuentra embebido en las TIC, hoy sucedidas por las llamadas Tecnologías Emergentes (TE), y aquellas reconocidas también como Tecnologías para el cambio (TC).

\section{Socio-cibernética: precursores y conceptualización}

La Socio-cibernética se reconoce como una rama de la Sociología. Se entiende como una disciplina en evolución, tan dinámica como la sociedad misma. A criterio de sus precursores, la Socio-cibernética involucra la aplicación de conceptos, métodos e ideas de lo que se ha llamado nueva Cibernética o "Cibernética de segundo orden" en el estudio de sistemas sociales y culturales (Geyer 2006). Es la disciplina que se encarga de aplicar la cibernética a lo social. Es una manera de entender las ciencias sociales y un modo de proceder a la hora de aplicar el quehacer científico a las cuestiones sociales.

El origen etimológico de la Socio-cibernética expresa la conjunción de dos conceptos básicos: la raíz "socio" que denota todo lo referente al mundo de lo social, y el segundo, el de "cibernética" en este particular se restablece tres momentos de contacto:

a) la acepción propia del término griego "kybernetes" para hacer referencia a la ciencia o arte con capacidad de guiar, orientar, intervenir las sociedades. Para (Geyer 2006) la atención debe estar dada en el hecho en que tales sociedades no son necesariamente de orden jerárquico. 
b) la acepción propia del término en la dimensión de Cibernética según el uso dado por Ampere.

c) la acepción propia del término en la dimensión y propuesta sistémica de Nobert Wiener.

Al respecto, se coincide con (Scott 2006) quien observa que históricamente, la Teoría de Sistemas y la Cibernética se desarrollaron en diferentes contextos. Se comparte el criterio que la formación y desarrollo de la Socio-cibernética como disciplina sigue los principios básicos de la Teoría de sistemas y de la Cibernética de comienzos del siglo XX pero sin desconocer sus antecedentes originarios.

Además, se reconoce la importancia de la contribución e influencia ejercida por las teorías de Niklas Luhmann (Paetau 2006) y de Walter (Buckley 1967). Este último se considera un pionero en aplicar la perspectiva de los conceptos de la teoría de sistemas a los sistemas sociales, al sostener que la naturaleza específica de los sistemas sociales es lo que los hace ser unidades viables de análisis y útiles para la construcción de teorías.

Conviene apuntar que la obra de Norbert Wiener reconoce la Teoría de Sistemas como parte de la Cibernética; tendencia que se mantiene prácticamente en toda la literatura científica posterior. Se considera que es un precursor directo de la Socio-cibernética a partir de los postulados que expresó en su libro "Cybernetics. Or control and Comunication in the animal and the machine" publicado en 1948.

También, se considera como un precursor práctico de la Socio-cibernética a Stanford Beer, quién en toda su obra proporciona una clara aplicación de la Cibernética al análisis de sistemas sociales y desarrollo de soluciones para problemas de desarrollo nacional y otras unidades, mediante lo que él definió como Modelo de Sistemas Viables (Beer 1975). De alta relevancia para la disciplina es la noción de Beer de considerar el cambio social planificado, como una herramienta para mejorar las condiciones de vida de todos los miembros de un sistema social (Beer 1972, 1975).

Un intento de aplicación del Modelo de Sistema Viable tuvo lugar en Chile a comienzos de los años 1970, liderado por el Presidente Salvador Allende, con el propósito de elaborar, en tiempo real, información y herramientas que facilitaran la toma de decisiones para la economía e industrias del país y ampliar la participación ciudadana en el gobierno.

Se considera que el proyecto Cybersyn, nombre derivado de la combinación de las palabras "cibernética" y "sinergia", constituye un ejemplo válido para ser estudiado como caso de estudio. Por eso en el epígrafe siguiente dedicado a la relación Socio-cibernética y desarrollo social se amplía su referencia. 
En fecha más reciente, se sugiere que la Socio-cibernética (Mulej 2006) tiene como propósito conocer y comprender las complejas relaciones humanas y sociales. Mientras que (Menanteau-Horta, Marcuello-Servós 2006) estiman que "la tradición establecida por la Teoría General de Sistemas, conocida también por el nombre de Socio-cibernética, puede aportar una perspectiva más dinámica y más completa tanto para las actividades profesionales como para las preocupaciones teóricas de las ciencias sociales."

El desafío principal de este marco de referencia es utilizar las coordenadas de la teoría general de sistemas en el análisis de la conducta y organización social. $\mathrm{Al}$ hacerlo, hay que tener en cuenta que aparecen problemas y paradojas tales como la que hay en las relaciones de la observación-observador-observado, el control y la evolución de sistemas que se auto-controlan, o la planificación en sistemas sociales donde las variables que se consideran no se alcanzan a abarcar en forma completa, ni por mucho que se amplíe el conocimiento de ellas sirven para un mejor control.

La idea que el paradigma del control del sistema se puede aplicar a la conducta y organización social definidas como sistemas sociales, representa entonces un paso significativo para aceptar la noción de planificación y acción social, de crítica e intervención social en tareas, funciones y servicios necesarios para el desarrollo social (Menanteau-Horta 2006).

Otro ejemplo de transferencia teórica, con la cual la Socio-cibernética aspira a incrementar el conocimiento sobre los sistemas sociales, está ilustrado por el concepto de autopoiesis desarrollado por Maturana y Varela (1972, 2000, 2002), en el campo de la Biología, e incorporado al terreno de las ciencias sociales por Niklas Luhmann $(1986,1998)$.

De acuerdo con Luhmann, el proceso de comunicación se erige como una unidad central de los sistemas sociales siendo la acción social subsidiaria de los procesos de comunicación, los cuales constituyen el núcleo autopoiético de todo sistema social. Esta observación tiene importantes repercusiones epistemológicas distinguiendo al observador de lo observado y, de modo más especial, pone en alerta a las teorías preocupadas de la causalidad tradicional de los riesgos originados por los problemas de la circularidad del sistema.

Respecto a lo anterior conviene señalar que el científico social es parte de aquello que observa. No es un gestor de conocimiento aséptico y externo al mundo como observador que se declara neutral. No puede serlo. La estrecha interdependencia entre el científico como observador y el objeto observado es lo que emerge en el proceso de auto-referencia que representa una de las claves sobre las cuales está desarrollando la Socio-cibernética actual, que sostiene que los sistemas sociales entendidos a partir de las claves y conceptos mencionados 
remiten a una revisión del conocimiento social necesario para estudiar la sociedad.

Todo conocimiento sobre la sociedad se retorna al sistema social de forma que varía las propias estructuras del sistema e incluso su comportamiento. Esta retroalimentación del sistema configura al sistema y las observaciones o mediciones que se hacen del mismo. Los modelos sociales con los que se justifican investigaciones o políticas de intervención social operan sobre la base de la construcción de predicciones respecto al sistema y en la validación de los resultados científicos (Bernd 2006).

Las reacciones ante esta retroalimentación configuran las interpretaciones de los actores sociales dentro del sistema. Así, el conocimiento del sistema social, sus estructuras y comportamientos están relacionados de manera que al actuar sobre uno de ellos, se reorganiza el sistema en su conjunto. Son, por lo tanto, procesos de adaptación y aprendizaje, que exceden tanto al actor social como al científico.

Además de esto, los principios conceptuales de la "cibernética clásica" contribuyeron también a despertar el interés de la sociología y de otras ciencias sociales en el estudio de la teoría general de sistemas, y prestar atención a la creciente complejidad del cambio y desarrollo de los sistemas sociales. Todo esto después se concreta en procesos e interacciones sociales con consecuencias directas en todas las áreas de lo social, lo económico y lo político, por ende en el Derecho.

En fecha tan temprana como el año 1948, quedó establecida la relación de la Cibernética con el Derecho dando origen a un área de conocimiento y desarrollo que mostraba una doble relación de objeto y medio; nace así lo que se conoce como Informática Jurídica y el llamado Derecho Informático el que en coincidencia con algunos especialistas debe ser visto cada vez más como Derecho de Alta Tecnología y preferiblemente como el Derecho de la Sociedad Tecnológicamente avanzada, ese que es el resultado y expresión de la sociedad actual (Martino 2002, Suñé 2016).

En consecuencia, se reconoce y acepta que hay una vanguardia jurídica que considera que se trata de todo el Derecho el que debe evolucionar y continuar desempeñando su rol de organizador social en el Ciberespacio que es un escenario en otra dimensión pero es también una extensión del mundo real en el que se desarrollan relaciones sociales con implicaciones jurídicas. Por tal motivo, se considera que pretender encasillarlo en una rama del Derecho, es afiliarse a una visión reduccionista del fenómeno y caer en posiciones dogmáticas.

No obstante, desde el punto de vista práctico se reconoce la especialización en el ejercicio de la profesión por ende completamente compatible con la 
existencia de servicios legales especializados en Derecho Informático o la práctica de ejercicio de abogacía digital.

También se afirma que hoy los nexos que provoca la aludida relación de objeto y medio de las TIC con el Derecho se mantienen entre luces y sombras lo que se expresa en una dimensión incompleta de las vistas de organización, dirección y control de la sociedad tecnológicamente avanzada y peor aún la discontinuidad en la construcción de la llamada Sociedad de la Información y su hito de Sociedad del Conocimiento sobre bases de posiciones tecnocráticas en lugar de un enfoque socio-técnico. La crítica a la Teoría del "Informacionalismo" constituye también un ingrediente válido para los análisis desde la denominada Sociedad Red.

\section{Socio-cibernética y desarrollo social.}

La relación entre las funciones del desarrollo social y la Socio-cibernética está profundamente enraizada en la naturaleza de los procesos de cambio organizacional, mejoramiento de las condiciones de vida y la metodología cibernética. La noción de desarrollo social y la Cibernética surgen como nuevas perspectivas para la búsqueda de mejores formas y procedimientos para resolver problemas.

Aunque el concepto de desarrollo tiene una historia (Rist 2002) y una densidad de contenidos polisémicos y polémicos, sin embargo, se afirma, que cuando el desarrollo se define en términos de desarrollo social, implica la presencia de un diseño de intervención para mejorar las condiciones humanas. Esto es lo que permite diferenciar el concepto de desarrollo social como una herramienta para el bienestar individual y colectivo de otras formas de cambio evolutivo o de sólo crecimiento económico.

$\mathrm{Al}$ decir de Zamorano (2006), "el aprendizaje está relacionado con el proceso de resolver problemas" que equivale a decir que con el empleo de la metodología cibernética se puede descubrir, comprender y aprender acerca del mundo y de sistemas sociales. Por ende la Socio-cibernética, es una herramienta para asimilar realidades y esto debería ser, por cierto, un desafío común y algo compartido por la metodología de las ciencias sociales del siglo XXI.

La aplicación y relevancia de la Socio-cibernética en el campo de la acción social radica en las relaciones existentes entre los procesos de comunicación, intercambio de información y organización social. Estas relaciones permiten a los sistemas recibir y utilizar información lo que ayuda a que los sistemas sociales puedan cambiar y ajustarse al medio y condiciones externas. 
La creciente complejidad de la sociedad contemporánea incrementa aceleradamente la demanda de información y ello contribuye a hacer del rol de los investigadores sociales una función esencial para disponer de una comunicación transparente, una organización social justa, una acción colectiva eficiente y un cambio social adecuado (Martín-Barbero 2009).

Los procesos sociales y los problemas que afectan los sistemas sociales se caracterizan por ser dinámicos. Un mayor conocimiento de la sociedad y una mejor comprensión de las organizaciones en sistemas sociales complejos son fundamentalmente necesarios para la formulación de políticas públicas y la estructuración de programas nacionales e internacionales de desarrollo social.

El Proyecto Cybersyn, ha sido considerado el primer esfuerzo para implementar un programa de Socio-cibernética en América Latina y un referente válido para desarrollar aplicaciones de Gobierno Electrónico y de Gobierno de la Información en base a una visión socio-técnica de la informatización de procesos de administración y gobierno con apego a postulados infoéticos.

Sin embargo llama la atención las escasas publicaciones académicas al respecto lo que permite afirmar que es un proyecto desconocido. La pregunta es ¿por qué se habla poco del Proyecto Cybersyn a pesar que ha generado metodologías para compartir y al que se le reconoce como referente para comprender lo que hoy se conoce como Big Data?

Para contribuir a buscar la respuesta se comparten a los objetivos básicos y generales del modelo de Sistema Viable que se resumen en los principios operacionales siguientes:

(1) Incorporar la ciencia y la tecnología a los procesos de comunicación y mecanismos de decisión para facilitar la planificación, la organización y el desarrollo;

(2) Promover la comunicación continúa y transparente entre el gobierno y todos los miembros del sistema social;

(3) Aplicar una metodología que permita la comunicación entre todos los sectores del sistema para hacer posible la organización y el aprendizaje sociales tendientes a resolver los problemas y desafíos del desarrollo social;

(4) Facilitar y estimular la participación social que refleje plenamente las necesidades y la voluntad de las personas, desde la participación ciudadana.

(5) Promover el desarrollo social por ser éste un mecanismo funcional esencial para el mejoramiento del bienestar humano.

Asimismo, se declaran los tres conceptos motores fundamentales del proyecto para atender la complejidad del sistema chileno: el de viabilidad, el de recursión y el de autonomía. 
Estos principios fueron incorporados dentro de un modelo neuro-cibernético basado en la idea de que el manejo de una organización o sistema es una función de control, orientación y guía.

Aunque la experiencia de este proyecto en Chile fue breve y terminó abruptamente con el golpe militar de 1973, se reconoce que los objetivos y componentes principales de esta iniciativa tienen un merecido valor conceptual y ofrecen un importante aporte intelectual y práctico para quienes tienen interés en el estudio de los problemas sociales y las posibles herramientas para extirparlos. Encierra además un valor para el estudio sobre la experiencia puede contribuir a asumir posiciones propias que ayuden a superar el estatus de consumidores de tecnologías a usuarios inteligentes de modelos tecnológicos con fines sociales.

En décadas recientes el Modelo de Sistemas Viables ha capturado el interés académico de la Sociología, Ciencias Políticas, Relaciones Internacionales y también su uso práctico por profesionales de otras disciplinas para atender problemas usualmente encontrados en la administración de empresas, en la organización industrial y en los cambios necesarios para el desarrollo de las naciones. El Modelo de Sistemas Viables es un recurso para gestionar la complejidad.

Espejo y Harnden (1989) describen la aplicación de este modelo a problemas específicos en las áreas del cambio social y desarrollo. Beer (1989), por su parte, informa la utilización del Modelo de Sistemas Viables en el campo internacional incluyendo referencias a experiencias desarrolladas en Inglaterra, Canadá, Europa, Estados Unidos, Australia, Nueva Zelanda, Chile y en otros países de América Latina.

La naturaleza recursiva de los sistemas sociales permite, y aún requiere, que se reconozca la importancia de cada individuo en cuanto es una persona humana dentro de un sistema. Esta condición de sistema lleva consigo la aceptación de valores ético-sociales que usualmente se observan conectados a un tipo de cambio social con propósito o desarrollo.

Según Maturana y Varela (1988), la dimensión axiológica de los seres humanos es clara al considerar que "En el hombre como un ser social, todas sus acciones, aún aquellas individuales como expresiones de preferencias o rechazos, afectan constitutivamente las vidas de otros seres humanos, y por eso, tienen un significado ético."

Por eso, a continuación, se aborda otra arista del desarrollo social en vinculación con la Infoética y el Derecho. 


\section{Infoética, Socio-cibernética y desafíos del desarrollo social internacional. Contribución desde el Derecho}

Los objetivos y metas de la Infoética están orientados a que los poderes públicos garanticen el acceso a la información con acciones concretas que permitan ampliar y hacer más igualitario el acceso a la información.

En síntesis se puede decir que desde la UNESCO se reclama a los Estados miembros a que se preste especial interés en lograr un mayor acceso a la información de dominio público en un contexto mundial; concebir estrategias para superar los obstáculos económicos al acceso; potenciar el derecho a comunicar y el reconocimiento de uso legítimo de la información así como la utilización eficaz de las telecomunicaciones y de la circulación de la información en la educación, la ciencia y la cultura.

Los Estados miembros para satisfacer los objetivos y alcanzar las metas se encuentran convocados a la definición de las normas que han de regir en términos de derechos, deberes y responsabilidades. Especial atención debe tener el reconocimiento de la aplicación de excepciones legales a los derechos de autor con el fin de facilitar el acceso a la información de los países en desarrollo.

Otro elemento regulativo a tener en cuenta lo constituyen las normas de estandarización. La vía de la estandarización técnica pasa por la armonización legislativa y la cooperación política. Las organizaciones de servicios y la empresa moderna deben ser ayudadas a competir en todo el mundo.

Por otra parte, la realidad nos muestra elementos que no pueden ser desconocidos: la existencia de derechos supraestatales como la Unión Europea o la Corte internacional de Justicia; la fuerza económica transnacional de un mundo de la producción y el consumo siempre más globalizado; el avance tecnológico de los medios de comunicación de masa que constituye una opinión pública global y que hace saltar las referencias espaciales y temporales del pasado.

En coincidencia con (Martino 2015), se afirma que la globalización no procede a través de formas claras de tradición del poder de las manos de algunos sujetos a otros, sino más bien acoyundados a las formas tradicionales nuevos sujetos y mecanismos se contribuye a roer y tal vez vaciar el poder establecido. El desafío a los Estados es más bien de tipo competitivo frente a iniciativas que les va quitando partes más o menos importantes del poder normativo, fiscal, jurisdiccional, de control de la moneda y hasta del territorio y del uso legítimo de la fuerza.

El presente muestra como nacen nuevos poderes que imponen sus reglas y concitan a los Estados a declinar en sus funciones. El Estado no solo ve 
disminuida una parte cuantitativa de su potencia, sino que las nuevas dinámicas de poder se transforman y se delinean nuevas expresiones de influencia, nuevos lazos y nexos de legitimación que se instauran entre los detentores del poder y los destinatarios.

El Foro de Gobernanza de Internet puede ser tomado como un ejemplo. La Gobernanza se gobierna desde la puesta en práctica de un Modelo de partes interesadas. Se afirma la noción de pluralismo de influencia o constelación o red de poder. El Foro Social de Internet se erige también como una vía alternativa.

En consecuencia se afirma que en el Ciberespacio operan nuevos sujetos políticos tanto públicos como privados, algunos informales, económicos $\mathrm{y}$ benéficos. Son ellos las grandes empresas transnacionales, las firmas de abogados, organismos supranacionales, sujetos regionales o provinciales, organizaciones no gubernamentales.

No obstante, el Estado sigue siendo el principal actor político nacional, aunque el privilegio de su territorio es al mismo tiempo un límite con respecto a otras organizaciones transnacionales.

De manera que se configuran las nuevas tipologías de poder con efecto transnacional. Las relaciones de derecho público enfrentan el auge del derecho privado. Se configura también un nuevo espacio de poder en su conjunto que se valoriza a partir de las formas de participación, la transparencia y la eficiencia. La gobernanza que cada vez se aleja más de la noción de gobernabilidad.

Hay una tensión permanente entre las sofisticaciones para individualizar al sujeto en función de la seguridad y los cuidados que deben ponerse en no desvirtuar la intimidad y el ejercicio de libertades. Ello es una manifestación de los efectos horizontales de los derechos fundamentales.

La Infoética es punto de partida para la toma de decisiones que garanticen la protección de la dignidad humana en la era digital. Constituye una convocatoria para la adopción por los poderes públicos, las empresas y la sociedad civil, de estrategias tendentes a garantizar la seguridad, la protección de la vida privada y la libertad de expresión sobre las redes mundiales de información, incluidas: proposiciones para la definición y la aplicación de códigos de conducta que aseguren la fiabilidad y la seguridad sobre las redes mundiales.

Conocer sus postulados también favorece la formulación de principios directores de autorregulación que garanticen un equilibrio razonable entre el derecho de los usuarios a la libertad de expresión y el derecho a la vida privada.

El desarrollo social con auxilio de la metodología jurídica integrada a las tecnologías emergentes recorre todo lo que está cambiando en nuestras vidas gracias a las tecnologías desde mutaciones de hábitos sociales hasta una diferente relación con los otros y con la administración pública. 
Las TIC han favorecido la comunicación instantánea y la globalización. Estar dentro de la red significa estar vinculado desde cualquier lugar del mundo con todos los otros. La diferencia de latitudes y longitudes se anula en el tiempo de la comunicación. Las TIC y los bienes que de ella se derivan son considerados elementos clave para el desarrollo. La economía mundial es digital (CEPAL).

Las redes sociales, con virtudes y peligros, como es típico en una praxis social multitudinaria son la manifestación del cambio en las relaciones de comunicación y la expresión de la noción de red que caracteriza la sociedad actual (Martino 2015).

Este cambio en la forma de establecer relaciones entre los individuos se manifiesta también entre los individuos y el Estado, entre los Estados, entre los particulares con las empresas, de las empresas entre sí y con el Estado. Es una expresión de relación de todos con todos.

La introducción de las tecnologías en los procesos de gestión del Estado que permitió instaurar el Gobierno Electrónico comprende el auxilio tecnológico para la toma de decisiones, la gestión de datos y la distribución en su modo de hacer y por ende otro modo de interacción entre sujetos.

El Gobierno Electrónico si bien amplia la capacidad para la resolución de los problemas lo más cerca posible al lugar donde se originan para su funcionamiento se requiere que se cumplan con dos condiciones: que existan redes confiables para que el interesado y el funcionario puedan actuar y que el personal esté capacitado.

La capacitación es una relación relevante. Es necesario que todos estén preparados para asimilar la transformación. Hay que generar capacidad para enfrentar los nuevos desafíos y ello implica asumir otras formas de enfrentar y resolver problemas, se exige un pensamiento sistémico. Se exigen modelos de servicios y negocios responsables.

El Gobierno Electrónico como el Comercio Electrónico cambia nuestra manera de enfrentar el desarrollo social pero también sienta las bases para favorecer el cruce de datos y obtener un perfil más afinado de cada ciudadano en uno del consumidor en el otro.

Con los procesos de informatización cambian las formas de validar la autenticidad de una persona o de un documento. La aparición de la firma digital es una revolución extraordinaria que modifica las formas de prestar consentimiento en cuanto a la forma, el lugar, la ley aplicable.

A la vez exige evaluar las consecuencias jurídicas y tecnológicas de una técnica que sigue en permanentemente desarrollo y generalización en medios de comunicación y gestión de información. Para su implantación se necesita de 
políticas públicas y herramientas bien concebidas para satisfacer la demanda de todos elementos implicados.

El cambio en las tecnologías incide en los hábitos y en los valores. Por eso es necesario conocer los postulados de la Infoética porque pone a los individuos y a las sociedades frente a hechos que antes no habían podido acaecer y que hoy, acaeciendo, tratan en modo diverso tanto la ética cuanto la moral social.

Cambia la percepción social ante la práctica de algunas conductas que siendo ilícitos jurídicos se aceptan como válidas por constituir una especie de justicia por mano propia. El desarrollo y distribución de programas malignos y los llamados virus informáticos pueden ser tomados como ejemplo (Amoroso 1999).

La existencia de dinero electrónico no solo pasa por los bitcoins sino por la aceptación de algunos Estados de tal tipo de moneda, esto también cambia las relaciones comerciales y necesita de una regulación jurídica: es un hecho nuevo (Martino 2015).

El cambio de las relaciones de valores y su expresión ética, son problemas de alta complejidad y para su comprensión se reclama de una visión sistémica.

La Socio-cibernética puede aportar los medios para el diseño, la implementación y la necesaria evaluación de impacto para el desarrollo de soluciones de la agenda digital de los países y su proyección ante los objetivos y metas de desarrollo social 2030.

Como se ha mencionado, en la sociedad tecnológicamente avanzada, reconocida indistintamente como Sociedad de la Información o Sociedad del Conocimiento, e incluso algunos la identifican como Sociedad Red, o simplemente Ciberespacio, la Socio-cibernética puede ser una vía para buscar soluciones para enfrentar y ordenar procesos destinados a solventar algunos de los males que se ha generado por la incidencia social de las TIC y las TE.

El primer paso para comprender el fenómeno es reconocer una convergencia de dos escenarios: el "mundo real" en que la realidad cotidiana de cualquier ser humano u organización puede ser extendida al "mundo virtual" ese espacio informático denominado Ciberespacio, en el que se interactúa con información digital y en el que las nociones jurídicas de espacio, tiempo y cosas han cambiado para el Derecho (Martino 2015), y hoy se manifiestan en otra dimensión. Hay que estar preparados porque la naturaleza disruptiva de tecnologías emergentes promete más cambios.

Al decir del Dr. Vittorio Frosini "(...) Esta es la nueva forma de la información, que se asimila en nuestro tiempo de civilización tecnológica, después de las formas anteriores de información gestual o verbal, simbólica con dibujos y con escritura, y más tarde con la imprenta y con los medios de transmisión eléctrica, hasta llegar al actual tratamiento (...)" (Frosini 1978), en 
este caso la información digital en virtud de la cual la humanidad ha ido identificando un cambio en los paradigmas en cuanto al soporte informacional.

También crece la convergencia en los medios de transmisión de información y soportes a través de los cuáles se almacena, procesa y trasmiten diferentes tipos de información (texto, imagen y sonido) y a partir de las cuáles se genera el mensaje e implica cambio en los modelos de gestación y consumo de la comunicación (Aguado 2006).

Así el desarrollo de la infraestructura mundial de información ha transformado nuestro entorno común, especialmente en lo que se refiere a la generación y transmisión de datos, información y conocimiento, convirtiéndose a su vez en generador de nuevas fuentes y formas de realización de empleo, por ende trasciende a nuestra vida cotidiana.

El paradigma papel hoy convive con el paradigma digital, pudiendo ser gestionado en sistemas híbridos de información. La documentación soportada en papel puede ser convertida con facilidad en objetos digitales derivados y ser gestionados para su preservación y consecuentemente su acceso como mismo sucede con los objetos digitales originarios, unos y otros integran al Patrimonio Digital.

Cada día la información se consolida como bien social, económico y jurídico autónomo. Se le reconoce valor como materia prima fundamental en el cuarto sector industrial. Se identifica además, como un recurso estratégico para el desarrollo, se afirma que la economía mundial es digital (CEPAL 2016); de manera tal, que los cambios estructurales son palpables en términos de indicadores de crecimiento económico, por ello la distinción entre países inforricos e infopobres, lo que es también una de las vistas para el análisis de la brecha digital y del derecho al desarrollo.

También se reconoce, que la aplicación de las nuevas tecnologías de la información y la comunicación al entorno social en general es fuente de un sector productivo en tanto genera bienes y servicios y modos diferentes de realización del comercio internacional (Paredes 2008).

Hoy en virtud de la llamada economía digital se afirma que es también una fuente que genera fuente de empleo laboral diferente a los tradicionalmente reconocidos, aunque se advierte también como otra vista de la brecha digital en este caso referido a capital social y a la capacidad de conocimiento instalada.

A nivel social, surgen oportunidades sin precedentes para la comunicación (Córdula 2010) lo cual favorece extraordinariamente procesos de generación e intercambio de información. Pero, si se reconoce que el Ciberespacio es un espacio de todos, como se ha dado en decir, se debe hacer de él también un espacio de la democracia multicultural y del multilingüismo. 
El Ciberespacio es un ámbito para el ejercicio de libertades y derechos pero es también un espacio de responsabilidades que se comparte como espacio cívico.

Por otra parte, también se señala que el empleo razonable de grandes sistemas de información en interés de la sociedad, así como la protección segura de toda la información son factores determinantes de una actitud de respeto ante las posibilidades que brindan las tecnologías de la información y la comunicación, y éste debe también considerarse como axioma ético.

Desde la UNESCO se considera que la adopción de directivas éticas coherentes es esencial para la construcción de la Sociedad del Conocimiento más inclusivas y participativas, por ello impulsa y reconoce como una meta la definición y adopción voluntaria de directivas éticos para profesionales de los medios, los productores de información y los proveedores de servicio, entre otros actores del Ciberespacio.

La Infoética propone un conjunto de principios y directivas éticas que sostienen los valores de: la libertad, la igualdad, la solidaridad, la tolerancia y la responsabilidad compartida. Acogerse a los principios de la Infoética y practicarlos, contribuirá a fomentar las relaciones de confianza y gestión de la seguridad en la era digital. Se estima que al elevar la sensibilidad sobre los principios del deber ser se puede contribuir a fomentar el respeto a la dignidad humana en el Ciberespacio (Quéau 2010).

Entre los componentes que integran la estructura global de la información se reconoce a la información que es el elemento estratégico y a la infraestructura material de conexión e interoperabilidad que incluye también al software. También se considera de modo muy especial al actor humano, dado que es capaz de producir los objetos indispensables a través de los cuales se materializa el Ciberespacio; es el sujeto activo de esta realidad. Todo ello deriva en consideraciones ético-jurídicas sobre el tratamiento digital, uso y la conservación de la información.

Los enfoques sociocibernéticos en los proyectos de informatización deben facilitar el diseño de los mismos, en términos de conexión e interoperabilidad, en correspondencia con las buenas prácticas en la gestión de datos enlazados para favorecer la necesaria transparencia en los procesos de la Administración (Cerrillo-i-Martínez 2012), que es una meta de la Infoética para garantizar el acceso al conocimiento y aumentar las posibilidades de participación democrática en la gestión de gobierno.

Las TIC están implicando un cambio significativo en los mecanismos a través de los que se canaliza la transparencia administrativa. Las TIC permiten a los ciudadanos participar activamente difundiendo información generada a partir 
de datos en formatos abiertos y procesables automáticamente, interactuar entre ellos y con las administraciones públicas así como personalizar la información del sector público. Además, las TIC incrementan las finalidades de la transparencia al convertirse en un mecanismo que permite una mayor participación de los ciudadanos en las administraciones públicas y un mayor desarrollo económico y social.

Sin embargo, para que estos efectos sean reales y para mejorar efectivamente la transparencia de las administraciones públicas es necesario regular el impacto de las TIC en los diferentes mecanismos e impulsar un cambio de la cultura administrativa. Así pues, el propio concepto de transparencia puede verse afectado por las TIC.

Es necesario repensar la enseñanza del Derecho como ciencia social a partir de la doble relación que tiene con las TIC, en tanto medio y objeto, así como las interacciones con otras ciencias y áreas de conocimientos que la tecnología impone como fenómeno cultural. Es necesario recurrir a la práctica de nuevos principios de interpretación y formación de normas jurídicas; ello demanda una articulación de valores, derechos, deberes y responsabilidades tecnológicas, una nueva cultura jurídica (Pérez Luño 2014).

Dos posiciones prevalecen en cuanto a la potencialidad del Derecho como instrumento regulador de las situaciones a que da lugar la incidencia social de las tecnologías de la información y las comunicaciones, y especialmente el fenómeno de las redes de alcance global asociado al Ciberespacio.

La primera de ellas, encabezada por la conocida frase de "nada nuevo hay bajo el sol", asevera la suficiencia de los instrumentos jurídicos clásicos preexistentes a la Era Digital para brindar solución.

La segunda posición, sostiene que los instrumentos jurídicos tradicionales resultan inoperantes para resolver situaciones generadas dado el carácter novedoso de las situaciones a que dan lugar las realidades tecnológicas, propias del ámbito natural del fenómeno en el Ciberespacio.

El Derecho es el instrumento orientado a preservar la dignidad humana en la Era digital y el medio institucional de regulación de las relaciones humanas, dado que donde hay sociedad hay derecho. Tal afirmación se ampara en los Principios Generales de Derecho que son la piedra angular para erigir todo marco legal y son el fundamento para determinar aquellas reglas que se integran como ordenamiento jurídico. Constituyen también la base para identificar y comprender fenómenos nuevos y proveer soluciones expresadas en forma de derecho positivo, ejemplo de ello lo constituyó reconocer las reglas aplicables de la contratación a distancia y entre ausentes y así encontrar fundamento teóricopráctico para ordenar la contratación electrónica. 
También su aplicación a casos no previstos por la ley puede contribuir de guía orientadora de la interpretación en función de resolver situaciones que las normas no regulan implícitamente, pero que reclaman tutela jurídica.

Así se comportó respecto a la protección de legal del software para decir que le resultaba aplicable instituciones del Derecho de autor como obra literaria cuando realmente no lo es, la mirada estuvo centrada en que software es documentación y en ella está contenida el conocimiento que sirvió de base para su desarrollo como son los documentos técnicos que expresan el diseño y arquitectura de la solución, así como lo que resultó como producto final expresado en manuales de uso por solo citar un simple ejemplo.

Hoy, este debate sobre el tema de Software continúa alrededor de la Convención de Berna y la realidad demuestra que el Software requiere un régimen de protección sui géneris y que las soluciones adoptadas también tenían brechas. Mientras ha aumentado el número de patentes sobre soluciones informáticas a pesar que quedó expresamente excluido del régimen de propiedad industrial.

Curiosamente los titulares de dichas patentes son las grandes compañías de software lo que condiciona además el proceso comercial de licencias de uso tanto para el desarrollo de aplicaciones, como los procesos de despliegue y mantenimiento de las soluciones. También impacta a los usuarios finales. Queda pendiente evaluar si tiene relación con la llamada burbuja tecnológica.

De manera que la variante de articulación de formas clásicas de gestación de reglas constituye una mixtura reactiva que trae consecuencias también para Derecho en función de garantizar la legalidad y la seguridad jurídica desde el punto de vista de articulación del orden legal junto al diseño de lo que generalmente llamamos tecnología. La pregunta entonces es: ¿Qué Derecho es el que se necesita?

Se evidencia así un problema de profunda raíz política y requiere además de una meditada reflexión académica y de acciones prácticas, considerando los varios y diversos operadores.

Se debe actuar teniendo en cuenta los diversos escenarios en los que hay que desarrollar tales acciones para la toma de decisiones a favor del interés social y el bien común. Y aquí vuelve a revelarse el nexo con la Infoética y la Sociocibernética.

Sin conocer cuáles son los verdaderos dilemas que provoca la tecnología y si no se cuenta con modelos cibernéticos para evaluar impacto, los análisis pueden pecar de ser subjetivos y fruto de la improvisación, solo se consigue propiciar soluciones aparentemente válidas y en el fondo alternativas precarias que reproducen males y comprometen el desarrollo. 
Otro aspecto a tener en cuenta es la necesidad de generar instrumentos internacionales especialmente desde las organizaciones internacionales de carácter público como es el caso del sistema de órganos de Naciones Unidas sin desconocer mecanismos de consulta y participación que permitan multiplicar las miradas cuál ejercicio de política pública internacional. Para ello también es válido contar con datos objetivos. Se considera que el sector académico está llamado a jugar un papel socialmente activo para aportar los datos necesarios.

Para contribuir al debate se abordan algunas interrogantes que ayudan a evidenciar aristas del problema: ¿Los instrumentos jurídicos existentes (leyes, principios contractuales, procedimientos administrativos y jurisprudencia) son suficientes para propiciar el desarrollo armónico de los procesos tecnológicos y eficientes en cuanto a los avances en la tecnología de la información en general se refiere, y su producción y uso en particular?

En el contexto de los valores e intereses jurídicos generales no puede ser excluido el hecho de que los instrumentos existentes necesiten algunas modificaciones o ajustes. En una revisión del ordenamiento jurídico es muy probable que la norma, como tal, resulte ineficaz. Existen situaciones en las que Derecho tradicional no es suficiente; por tanto es necesario atemperar o llevar a cabo un proceso de modernización del Derecho, en su sentido más amplio, no solo de carácter práctico como por ejemplo el empleo de tecnologías, sino sobre todo en aspectos teóricos.

Se debe recordar que los problemas del Derecho son tanto teóricos como prácticos. Los nuevos paradigmas demandan respuestas innovadoras pero también el orden se funda en principios que rigen las relaciones que son objeto de tutela jurídica.

Puesto que el marco jurídico es anterior, es probable que la ley existente no pueda ser aplicada en todo o es al menos confusa con respecto a varios aspectos de producción y uso de tecnología de la información, pero no todo el Derecho es ineficaz.

Es importante insistir en que no se puede confundir la norma jurídica con el Derecho, esa es una posición reduccionista de éste. Ni todo lo que se necesita son las leyes, ni solo con leyes se resuelven los problemas, la ausencia de ley es a veces esgrimida para ocultar otros males o voluntades que quieren mantener su estatus.

El reclamado marco legal se debe componer de disposiciones jurídicas que constituyen un As de derechos. Es también necesario que existan normas técnicas que ordenen y rijan para procesos de desarrollo tecnológico. Otros aspectos son objeto de reglas contractuales. 
Es importante establecer códigos de deontológicos y mecanismos de retroalimentación que permitan que se conozca de los resultados de la solución de conflicto. Todo ello es lo que se denomina como As de derecho y conforma un modelo para pensar y gobernar las TIC. Este modelo es aplicable a nivel local e internacional. (Amoroso 2014).

Las condiciones económicas de la producción tecnológica parecen estar favorablemente influenciadas por instrumentos jurídicos existentes, como es el caso de las decisiones judiciales sobre el Derecho de Competencia.

En otros campos, la efectividad de instrumentos jurídicos muestra ciertas deficiencias, como sucede con la legislación civil sobre la estandarización de la calidad de los productos de las tecnologías de la información.

También, se evidencia en el tema de la responsabilidad o los instrumentos de Derecho Administrativo y Penal en cuanto a la vulnerabilidad y salvaguarda de la información se refiere. Aunque es importante resaltar que los valores protegidos siguen estando tutelados y el Derecho es capaz de dar una respuesta ante un quebrantamiento de la legalidad pero quizás la respuesta no se resulte eficaz.

También es cierto que algunos conceptos jurídicos tradicionales comienzan a resultar estrechos y es necesario responder ¿Cómo utilizar las nuevas tecnologías para propiciar la participación ciudadana y hacer de esta una herramienta emancipadora?; ¿Qué en materia de TIC tiene una función social?; ¿La información es un bien o una mercancía? ¿Debe permitirse el desarrollo de tecnologías de acuerdo a la "voluntad del mercado" o intentar programar las máquinas para salvaguardar los derechos humanos?

La información debe ser tratada en las diversas dimensiones que tiene y puede tener, tales como: un derecho, un documento y también como bien con valor estratégico. Tratarla exclusivamente como mercancía es un error.

La misma desempeña un papel muy importante en la educación, en la ciencia, en las relaciones sociales, en la representación de la realidad, en la preparación e implementación de fundamentos y decisiones. Por su importancia social e incidencia para el desarrollo, así como su significado económico, reclama un status legal sui géneris en las circunstancias más actuales.

Este estatus legal tiene que comprender los valores éticos y legales que les han sido reconocidos a sus titulares con anterioridad, de manera que lo que hay que comprender e identificar es el As de derechos que le tutelan en el entorno virtual. Conviene también advertir que lo virtual lo hace un espacio de bienes intangibles, y los bienes intangibles los ha reconocido y tutelado el Derecho antes de la incidencia social de las TIC. 
Por ello, la legislación de intereses relacionados con el tema no puede ser la visión de una sola norma para todo. Precisamente ante la necesidad de protegerla según sea el caso y acorde a las circunstancias que son producto de su valor polivalente es que tiene la información una relación derivada del sujeto que la genera y otra del receptor que la recibe. Difiere el caso de aquel que la manipula, procesa o conserva.

Por eso es que han proliferado las diferentes fórmulas relativas a una legislación especial para los temas de intimidad, privacidad, propiedad intelectual y las reglas para el intercambio electrónico de datos, por solo mostrar un ejemplo de la diversidad de ámbitos regulatorios que las relaciones emanadas de la generación y uso de la información digital puede generar.

Otro asunto que ha reclamado particular atención es la relación Redes y Derechos. Lo que generó una independencia relativa en el contexto del llamado Derecho de la Informática igual sucede hoy con el marco legal de la Computación en la Nube y pareciera que para cada evolución tecnológica dentro de las TIC da paso a una nueva especialización en el Derecho que no es más que reproducir el mismo error de reducirlo a solo normas jurídicas que hacen el marco regulador.

Una mirada unitaria del Derecho, es capaz de identificar de que en las redes se reproducen todos los problemas primarios objeto de estudio del Derecho, los que se pueden identificar metodológicamente en binomios de estudios, a saber: "redes y libertades; redes y propiedad; redes y responsabilidad; redes y fraude; redes y contratos y, más especial redes y seguridad" (Vivant 1995). Hoy se le suman los temas de redes sociales y más ampliamente Ciberespacio y relaciones sociales.

En sede del Ciberespacio hay que comprender la transformación de las nociones jurídicas de las categorías espacio y tiempo. Evaluar cómo ello impacta los principios sobre los cuáles descansa el principio de territorialidad y de la temporalidad, lo que es indispensable para la valoración adecuada del hecho jurídico. Como ya se apuntó, también cambia la noción jurídica de las cosas para el Derecho.

En base a ello, algunos alimentan la tendencia a declarar la "inoperabilidad" del Derecho para enfrentar y resolver problemas que se generan en las redes y el Ciberespacio. Tal postura constituye una incitación al caos ya que nada en la sociedad puede existir en estado de anomia y tanto las redes como el Ciberespacio es una dimensión de realización de las relaciones sociales y por ende de hecho jurídicos.

Por ello ante el auge de las redes sociales y todo aquello que conforma el Ciberespacio es indispensable la valoración adecuada de los problemas más 
acuciantes que han generado la desconfianza y la imposición de reglas muchas veces contrarias a los principios fundamentales de Derecho y el consenso internacional.

Cada día la jurisprudencia está demostrando su capacidad de aportar soluciones jurídicas, aunque las velocidades de respuesta deberían también estar en consonancia con las nuevas realidades.

Se coincide con Vivant en que (...) "Sobre las redes, una falsificación se mantiene una falsificación y una palabra de incitación al rencor racial se mantiene una palabra de incitación al rencor racial" (Vivant 1995). La acción y el daño es lo que tiene significación para exigir las responsabilidades de ahí la necesidad también de que las legislaciones penales perfeccionen la manera de identificar las conductas indebidas y especialmente que los países acuerden el catálogo actualizado de conductas de manera que sean expresivos de la voluntad de salvaguarda de la dignidad humana.

Más exactamente, es cierto que el Ciberespacio es un espacio de libertades pero también de responsabilidades -entre ellas las jurídicas-; y en el que los derechos tienen el límite que le impongan otros derechos, y en el que los valores e interés social tienen prioridad sobre los valores e intereses individuales.

Otros estudiosos como Jean Pierre Chaumoux, han abordado también el tema de los aspectos jurídicos de la información, todo lo cual ha dado origen a nuevas instituciones jurídicas o al planteamiento de reconsiderar algunas instituciones tradicionales del Derecho como el documento y los elementos constitutivos de su validez y valor probatorio, a partir de los nuevos soportes informacionales sobre los cuales se realiza la transmisión y la difusión de la información (Chaumoux 1980).

Pero esta realidad no debe ser analizada ajena a las diferentes circunstancias en las cuales surgen y se realizan estas instituciones jurídicas, dado el desigual desarrollo tecnológico del concierto de naciones, la ausencia de regímenes jurídicos internacionales y nacionales aplicables a las nuevas relaciones sociojurídicas, así como un desarrollo doctrinal dispar, todo lo cual trae aparejado relaciones de dependencia tecnológica y doctrinal, un proceso paulatino de transculturación y el cuestionamiento en cuanto a la validez del concepto de soberanía y la propia existencia de los estados-nación al identificarse un nuevo espacio sin fronteras y en el cual algunos vaticinan, como hemos descrito con anterioridad la inoperancia del Derecho.

Tales presupuestos si bien conducen acertadamente a una reevaluación y modernización del Derecho para su re-diseño, no dejan de ser una brecha también para imponer reglas de Derecho; pues la exaltada des-regulación como consecuencia de la incidencia social de las tecnologías de la información y la 
comunicación no es más que una forma de regular ajena al proceso actual de elaboración del Derecho y desconociendo realidades sociales y políticas diferentes, incorporando nuevas maneras de establecer reglas y a sus actores como titulares evitando incluso que los Estados participen de la toma de sus propias decisiones porque las mismas se adoptan desde la arquitectura y diseño de las aplicaciones informáticas.

El tema de los nombres de dominio aunque ha evolucionado, podría ser un buen ejemplo de lo que se ha planteado. No es éste un tema exclusivo del ámbito de la propiedad intelectual como se quiere ver en su relación comercial, es y mucho más preocupante porque es también un tema de soberanía sobre el cual hay mucho que reflexionar y proponer soluciones.

Por ello asumir la "inoperabilidad" Derecho o el "no-Derecho" para Ciberespacio, constituye un acto irresponsable, una incitación al caos. El Ciberespacio es una dimensión de realización de las relaciones sociales y por ende de hechos y actos jurídicos.

La imposición de reglas vía código informático también debe ser tenida en cuenta en este análisis. Es demostrable como en ocasiones esas declaraciones se esgrimen por conveniencia y en otras muchas para justificar o asumir una postura hegemónica en el gobierno y gobernanza de ese espacio informático porque lo cierto es que a la par de las discusiones y la dilación en la adopción de soluciones jurídicas con carácter internacional y vinculante, estamos regidos por un "Código legal" ese código que se ha ido creado y gobierna cual expresión de conquista silenciosa del espacio.

Por eso, se comparte el criterio de Lawrence Lessig de que "la razón de este enorme potencial de control sobre el Ciberespacio no sólo se encuentra en el poder legislativo del Estado, sino en la arquitectura (el código) de las nuevas tecnologías" (Lawrence 2006).

De manera que otro Derecho también existe, ese que está formado por reglas de facto y de jure, pero que están escritas en de 0 y 1 , y del que poco o nada se habla. Unas reglas que están implícitas en software y tecnologías que se consume, que imponen patrones de conductas y genera cambios de paradigmas, una nueva cultura.

Por ello, es de destacar en sentido general, que consustancialmente a este fenómeno desde el Derecho: prácticas judiciales, normas jurídicas y doctrina, se gesta un proceso de cambio en importantes instituciones jurídicas tradicionales y asistimos al surgimiento de otras nuevas devenidas de las emergentes relaciones socio-jurídicas generadas de la incidencia social de las nuevas tecnologías de la información y la comunicación, pero es importante propiciar la armonización en los procesos de elaboración legislativa y la solución de conflictos. 
Estamos asistiendo a la consolidación de nuevos usos y costumbres, y porque no decirlo a un nuevo "Derecho de Gentes", en todo caso, se trata de un complejo y acelerado proceso que debe avanzar bajo una premisa irrenunciable: construyámoslos entre todos.

La sociedad tecnológicamente avanzada que hoy se manifiesta en el Ciberespacio no convive ni conviene hacerlo en un espacio de "no derecho" por ello urge emprender acciones más coordinadas en el seno del Sistema de Naciones Unidas para la búsqueda de soluciones más efectivas y eficaces para el concierto de naciones cuál expresión de soberanía.

\section{Conclusiones}

La Socio-cibernética puede contribuir a identificar las variables de interconexión y ayudar a comprender el cambio dado que las posibilidades de los humanos y sus correspondientes tecnologías están orientándose hacia la realización de futuros deseados. También puede auxiliar a realizar estudios y brindar previsiones sobre el cambio para hacerlo más comprensible y más afín al desarrollo humano.

La Socio-cibernética en interacción con las ciencias sociales puede expandir su perspectiva de intervención y control de sistemas por la vía de la planificación social introduciendo la presencia y participación de múltiples actores en el sistema social.

Las ciencias sociales deben propender el desarrollo armónico para preservar la dignidad humana en condiciones de sostenibilidad pero necesitan empoderarse de nuevas herramientas.

Con auxilio de la Socio-cibernética las funciones de las ciencias sociales pueden contar un marco conceptual sólido y herramientas que les permita disponer de datos que le aportan una perspectiva válida y eficiente para brindar soluciones a un sistema social global y complejo.

Es necesario identificar los valores universalmente compatibles que sean expresados en forma de principios y conceptos jurídicos universales. Un enfoque sistémico e integrador que incluya la vista de la Infoética asumida como un estado práctico-evolutivo de la Ética permite identificar las relaciones relevantes con el Derecho.

La Socio-cibernética puede aportar en la evolución del Derecho a causa de la incidencia social de las tecnologías de la información y las comunicaciones, porque le puede dar un impulso revitalizador a la Informática Jurídica llenándola 
de nuevos contenidos que permitan identificar viejos problemas del Derecho y que fueron disueltos pero siguen sin resolverse a pesar del avance tecnológico.

Es necesario incidir con el ejercicio práctico desde aplicaciones Sociocibernéticas y establecer puntos de observación que catalicen los procesos de retroalimentación como sistema lo que permitiría a las ciencias sociales estar en condiciones de responder a interrogantes que necesitan de respuestas más inmediatas y eficientes.

La tecnología aporta protección por diseño y permite estandarizar patrones de diseño responsables. Un enfoque sociocibernético de los diseños pueden permitir alcanzar modelos de protección generalizables como estándares lo que permitiría también su evaluación de impacto. También puede favorecer la evolución del desarrollo de la herramienta y su integración a otros desarrollos.

Una lectura actualizada de la Informática Jurídica en base a un enfoque sociocibernético favorece el diseño de aplicaciones que se correspondan con el Principio de procedencia y orden natural de la gestión documental con el objetivo de favorecer la aplicación del Principio de Equivalencia funcional y su correspondencia el principio de Continuidad Digital que plantea la gestión y preservación del Patrimonio Digital.

Incluir los enfoques sociocibernéticos y enseñar su aplicación práctica en la enseñanza de las ingenierías informáticas, Derecho, Sociología, Ciencias de la Comunicación, Sicología entre otras a fines a las ciencias sociales y a los desarrollos tecnológicos ayudaría a la formación de un profesional con conciencia tecnológica y contribuiría a consolidar una práctica diferente en el diseño de generación de políticas públicas, reglas jurídicas y desarrollos tecnológicos.

\section{Referencias}

Aguado, Juan Miguel. Complejidad, cognición y comunicación: Sobre el contexto epistemológico de la Socio-cibernética. Libro Socio-cibernética. Lineamientos de un paradigma. pp. 81-124. Zaragoza. 2006.

Amozurrutia de María y Campos, José Antonio. Pensamiento sistémico y organización matricial orientados a la construcción de sistemas sociales: retos y herramientas ante la complejidad. Libro Socio-cibernética. Lineamientos de un paradigma, pp. 261-356. Zaragoza. 2006.

Amoroso Fernández, Yarina. Conversemos sobre un proyecto de eG e iG conocido por algunos e ignorado por todos. Conferencia Científica del Instituto 
Cibernética, Matemática y Física. Ministerio de Ciencia y Tecnología, diciembre 2014. Cuba.

Amoroso Fernández, Yarina. Virus informatici: aspetti legali. Informatica e diritto. Revista Internazionale dell Instituto per la documentazione giuridica del Consiglio Nazionale delle Ricerche. Florencia, Italia. 1999.

Bernd R. Hornung. El paradigma sociocibernético. Conceptos para la investigación de sistemas sociales complejos. Libro Socio-cibernética. Lineamientos de un paradigma. pp. 41-80. Zaragoza. 2006

Cerrillo-i-Martínez, Agustí. La contribución de las TIC a la mejora de la transparencia administrativa. ARBOR. Ciencia, Pensamiento y Cultura, Vol. 188 - 756, julio-agosto 2012.

Contreras, Fernando R. Cohesión social, comunicación y doble contingencia: la complejidad de la vida en sus múltiples posibilidades culturales. Libro Sociocibernética. Lineamientos de un paradigma, pp. 193-222. Zaragoza. 2006.

Córdula Almeida, Verbena. El derecho a la información y los nuevos paradigmas de la comunicación de masa. Revista Académica de la Federación Iberoamericana de Facultades de Comunicación Social. Diálogos de la comunicación, $\mathrm{n}^{\circ} 82$, septiembre - diciembre 2010.

Chaumoux, Jean Pierre: Consideraciones sobre la información. París. 1980.

Clair, Adam Les algorithmes: nouvelles formes de bureaucraties? http://internetactu.blog.lemonde.fr/2017/04/08/les-algorithmes-nouvellesformes-de-bureaucraties/ sitio visitado el día 9 de abril 2017.

Frosini, Vittorio: Cibernética, Derecho y Sociedad. Tecnos, Madrid, 1978.

Gallardo Garafulic, Rodrigo Daniel. El derecho de acceso a la información frente a la protección de datos personales. Santiago, Chile 2011.

Geyer, Felix. Reflexiones sobre el futuro de la Socio-cibernética. Libro Sociocibernética. Lineamientos de un paradigma. pp. 23-40. Zaragoza. 2006.

Marcuello, Chaime. La pregunta de Dorine, Libro Socio-cibernética. Lineamientos de un paradigma pp. 5-22. Zaragoza. 2006.

Martino, Antonio. Conferencia Magistral inaugural. IX Congreso Iberoamericano de Derecho e Informática. Costa Rica, 2002.

Martino, Antonio. Prólogo. Libro Sociocibérnetica, Informática Jurídica e Infoética. Buenas prácticas y Lecciones aprendidas. Editoriales UNIJURIS, Cuba. ASIDER, Perú. 2015.

Menanteau-Horta, Darío. Socio-cibernética aplicada al cambio social y desarrollo: el caso de Chile. pp. 389-406. Zaragoza. 2006.

Gómez Paidós, V. Sobre las aportaciones y límites de la Red y las redes sociales, para vertebrar nuevas formas de participación cívica en el espacio público. Barcelona, 2006. 
González, Jorge. Socio-cibernética y Cibercultur@: perspectivas, promesas y retos de diálogos interdisciplinarios. Libro Socio-cibernética. Lineamientos de un paradigma. pp. 407-448. Zaragoza. 2006.

Lawrence, Lessig. El Código 2.0. Edición original: The Code version 2.0, Cambridge, Basic Books, 2006.

Martín-Barbero, Jesús. De la sociedad regulada a la sociedad fragmentada. Conferencia magistral en el XIII Encuentro de FELAFACS, en La Habana, el 20 de octubre de 2009.

Menanteau-Horta, Darío y Chaime Marcuello-Servós: Una perspectiva sociológica para la acción social y el desarrollo: avances de la Socio-cibernética, 2006.

Nahabetián Brunet, Laura. Gobernanza y democracia. Hacia la construcción del gobierno de la información. Memorias de la IX Conferencia Internacional de Derecho e Informática de La Habana. Cuba. Mayo 2013.

Nápoles, Elena. Intervención especial. X Conferencia Internacional de Derecho e Informática de La Habana. Oficina Regional de la UNESCO. La Habana. Mayo. 2015.

Paredes, Ana Judith. Transformación de la cultura de servicios de información: una visión legal y de tecnologías. Revista Venezolana de Información, Tecnología y Conocimiento Año 5: No. 2, Mayo-Agosto 2008.

Paetau, Michael. Niklas Luhmann y la Cibernética. Libro Socio-cibernética. Lineamientos de un paradigma, pp. 161-192. Zaragoza. 2006.

Parra Luna, Francisco. El enfoque sistémico-axiológico en el estudio de lo social: sus posibilidades operativas. Libro Socio-cibernética. Lineamientos de un paradigma, pp. 223-260. Zaragoza. 2006.

Pérez Luño, Antonio Enrique. Los Derechos Humanos ante las Nuevas Tecnologías y Prólogo del Libro Nuevas Tecnologías y Derechos Humanos. El Tiempo de los Derechos 4. Editorial Tirant lo Blanch. Valencia, 2014.

Pintos, Juan-Luis y Marticorena Juan R. Análisis sociocibernético del discurso. La explotación de datos y los procedimientos informatizados en las investigaciones sobre Imaginarios Sociales. Un caso. Universidad de Santiago de Compostela. RIPS, Vol. 12, núm. 2, 2012, 47-79., España.

Quéau, Philippe. Cultura e Infoética. Una cultura capaz de civilizar la globalización. UNESCO, 2010.

Ramos Volonterio, Lourdes. Acceso a la información pública: normativa aprobada vs. realidad instalada. Construcción de ciudadanía y derechos encontrados. México. 2012

Rundle, Mary y Conley, Chris. Tecnologías Emergentes: Un estadio sobre sus consecuencias éticas. IFAP- Programa de Información para Todos. Sector de 
Comunicación e Información de la UNESCO. Paris, 2007.

Saarenpää Ahti. Interoperability and legal interoperability. Conferencia Magistral, Memorias de la X Conferencia Internacional de Derecho e Informática de La Habana. Cuba. Mayo 2015.

Sarlo Oscar. Internet: ¿instrumento axial o auxiliar en la investigación jurídica?, Conferencia Magistral, Memorias de la IX Conferencia Internacional de Derecho e Informática de La Habana. Cuba. Mayo 2013.

Scott, Bernard. Cibernética de segundo orden: una introducción histórica, pp. 125-146. Cor van Dijkum, El reto del pasado para el futuro de las Ciencias Sociales. Libro Socio-cibernética. Lineamientos de un paradigma. pp. 147-160. Zaragoza. 2006.

Suñé Llinás, Emilio. Prólogo de la publicación Bancos de Datos: pasado, presente y futuro. Universidad Complutense de Madrid, 2016.

ONU. 70a Asamblea General de la ONU. Documento Objetivos de Desarrollo Sostenible (ODS). Nueva York, septiembre de 2015.

Vivant, Michel: ¿Qué es el Derecho para las redes sin fronteras? Conferencia Magistral. Memorias del V Congreso Iberoamericano de Derecho e Informática, La Habana, 1996.

Wiener, Norbert: Cybernetics. Or control and Comunication in the animal and the machine. John Wily \& Sons. Nueva York. Año: 1948. 1ra. Edición.

UNESCO. Documentos oficiales de las Reuniones regionales y Congresos INFOETICA convocados por la UNESCO. Código de ética para la sociedad de la información, propuesto por el consejo intergubernamental del programa información para todos (pipt), 36 Conferencia General, Paris 2011

Zamorano, Héctor. La dinámica de sistemas y los modelos de simulación por computadora, Libro Socio-cibernética. Lineamientos de un paradigma pp. 357388. Zaragoza. 2006. 